\title{
Simulative Study of Different Control Concepts of Cooling System for Machine Tools
}

\author{
Linart Shabi $^{1}$, Juliane Weber ${ }^{1}$ and Jürgen Weber ${ }^{1}$ \\ ${ }^{1}$ Institute of Fluid Power, Chair of Fluid-Mechatronic Systems, 01062 Dresden, Germany
}

\begin{abstract}
Power losses in machine tools, e.g. during the standby, idle-, and manufacturing process, are converted into heat energy. This causes the machine frame and other machine components to heat up. As a result, the Tool Centre Point (TCP) of the machine tools is moved. The accuracy of the machine is thus reduced during manufacturing. The current cooling system design of machine tools is based on a centrally fixed pump supply that provides a constant cooling volume flow for cooling all the machine tool components. This does not correspond to the individual temperature development of the components, after all, the high temperature fluctuation arises and causes the thermoelastic deformation of machine tools. The main objective of this paper is to highlight the deficit of the current concept of cooling systems and to present a simulative study on the different controls concepts of cooling systems for machine tools. The results depict that the new concepts under consideration have a large potential for better thermal behaviour and lower hydraulic performance compared to the current cooling system design. The simulation results show a stability of the components' temperature profile as well as a decreased energy consumption of the cooling system.
\end{abstract}

\section{Introduction}

Over the last few years, energy-saving has become a more and more important topic and the public awareness of environmental issues has increased significantly. Using environmentally-friendly and energy-efficient products, much energy and many raw materials could be saved. In recent times, the development in the industrial sector has been focused strongly on producing in a more energy-efficient way.

In the field of manufacturing technology, machine tools are an essential part of a company's machine equipment. In addition to productivity, the demands on component accuracy and energy efficiency in production processes are also increasing [1]. During the production process, a part of the electrical energy is converted into heat energy and thermo-elastic deformations occur. These deformations affect the Tool Centre Point (TCP) position of the machine tools and lead to reduced accuracy. The heated components such as the rotary table, tool holders, linear guides etc. must be cooled. Therefore, fluidic systems such as cooling systems are installed to reduce the temperature fluctuations of the components. In order to reduce the thermo-elastic deformations that occur and to improve the manufacturing quality, it is necessary to minimize the heat input.

Previous research projects such as [2-4] mainly focused on the energy requirements of the machine tool and its main drives, thus reducing the energy consumption of auxiliary units by developing more efficient components and control strategies. The thermal behaviour, effectiveness, and design of the cooling system of a machine tool has not yet been described in detail. A detailed analysis of the existing cooling system structures and their effectiveness are therefore carried out in the subproject (A04) of the research project SFB/TR96, Thermo-energy design of machine tools. At the conclusion, the aim is to achieve an even temperature distribution and an efficiency increase of the machine tools.

The main target of this work is to show the deficit of the current cooling system structure by means of an experimental investigation carried out on a demonstration machine, and further, to present a simulative study of the different control concepts of cooling systems for machine tools.

The results illustrate that the new concepts being considered have great potential for improved thermal behaviour and lower hydraulic performance compared to the current cooling system design. The simulation results show a stable temperature profile of the components and a lower energy consumption of the cooling system.

\section{The current cooling system structure and its deficit}

The selected demonstration machine for the experimental investigation is type DMU80 eVo linear and is used mainly for high speed cutting (HSC). For more information about the demonstration machine see [5]. The cooling system of DMU80 cools 13 components 
simultaneously as shown in Fig. 1. The main function of a cooling system is to provide the cooling media for the components or spots of the machine to dissipate the heat energy and to avoid high temperature fluctuations within the machine structure. This helps to reduce the thermoelastic deformation and lastly increases the accuracy in the production process. Here, a centrally fixed pump 1 provides the cooling medium, $45 \mathrm{l} / \mathrm{min}$ at $4.5 \mathrm{bar}$, to motor spindle 2 , all the axis drives $(3,5,7,8)$ the housing of the $\mathrm{B}$ and $\mathrm{C}$ axes 4 as well as the rails of $\mathrm{X}, \mathrm{Y}$, and $\mathrm{Z}$ $(6,9-12)$. The determined hydraulic power of the central pump is about $340 \mathrm{~W}$. A mixture of water and $30 \%$ Glysantin ${ }^{\circledR}(\mathrm{G} 48 \AA)$ is used as cooling medium.

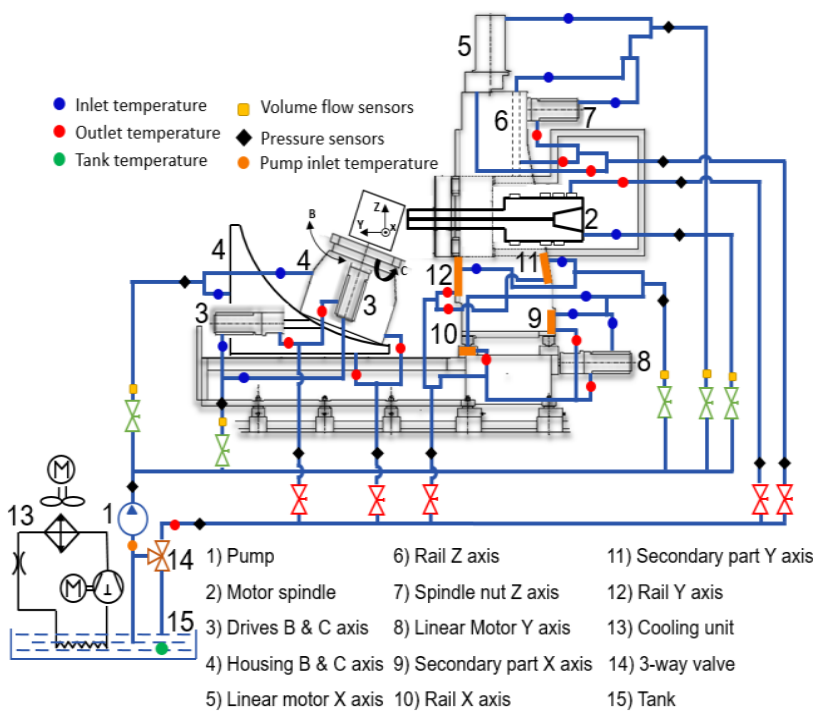

Figure 1. Cooling system structure of DMU80 eVo linear.

Moreover, cooling unit 13 of the DMU80's cooling system is not integrated in the return flow; it is mounted directly to the tank as bypass flow. Further-more, a threeway-valve 14 is placed into the return flow side. This valve is used as a diverting valve: with a defined setting, a part of the heated backflow is introduced directly to the inlet side of the pump and the remaining fluid flows back to the tank [6]. The controller of the three-way-valve adjusts the flow to the tank or to the inlet side of the pump so that the temperature on the pump inlet side always stays at approximately $25^{\circ} \mathrm{C}$, with only a minimal deviation [7].

To derive a statement about the thermal behaviour of the components to be cooled within the cooling system as well as to investigate the effectivity of the current cooling system, machine measurements of the cooling system of DMU80 with several sensors are carried out, as shown in Fig. 1,. The sensors used can help measure the temperature, pressure, and flow rate development. The measured process being considered for the experimental investigation is divided into four sub-processes: warm up process, idle process, set up process, and manufacturing process. The results of the idle- and the manufacturing process are shown in this paper. The idle process is based on the ISO 203-3 [8], where typical load cycles are considered and is carried out in a period of $830 \mathrm{~s}$. The manufacturing process is an exemplary process and is carried out in a period of $880 \mathrm{~s}$. Detailed information about the processes can be found in [9]. Fig. 2 and Fig. 3 show the cooling medium temperature development exemplified by the four components of the DMU80 during the idle- and the manufacturing process. The effect of the ineffective cooling of the components, a not demand-oriented supply, can been seen in the idle- and the manufacturing process. Over the entire idle and manufacturing process, the cooling mediums' inlet temperature of the drive $\mathrm{B}$ axis and spindle nut $\mathrm{Z}$ axis is higher than the outlet temperature. So the cooling medium is cooled while the components are warmed up. Other components, such as the motor spindle or the secondary part $\mathrm{X}$ axis, are cooled during the process.
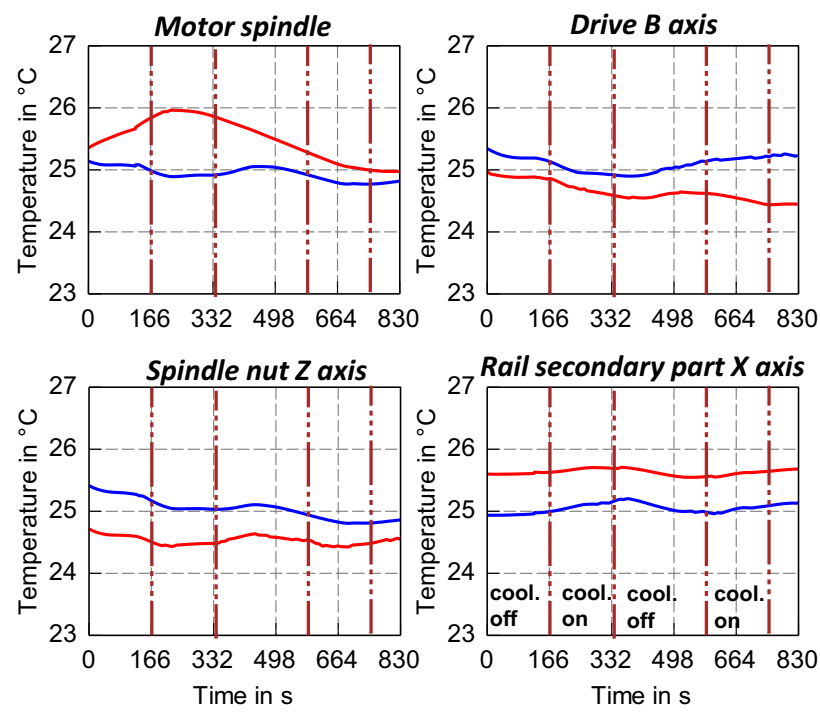

— Inlet temperature measurement

- Outlet temperature measurement

Figure 1. Temperature development in the idle process of DMU80.
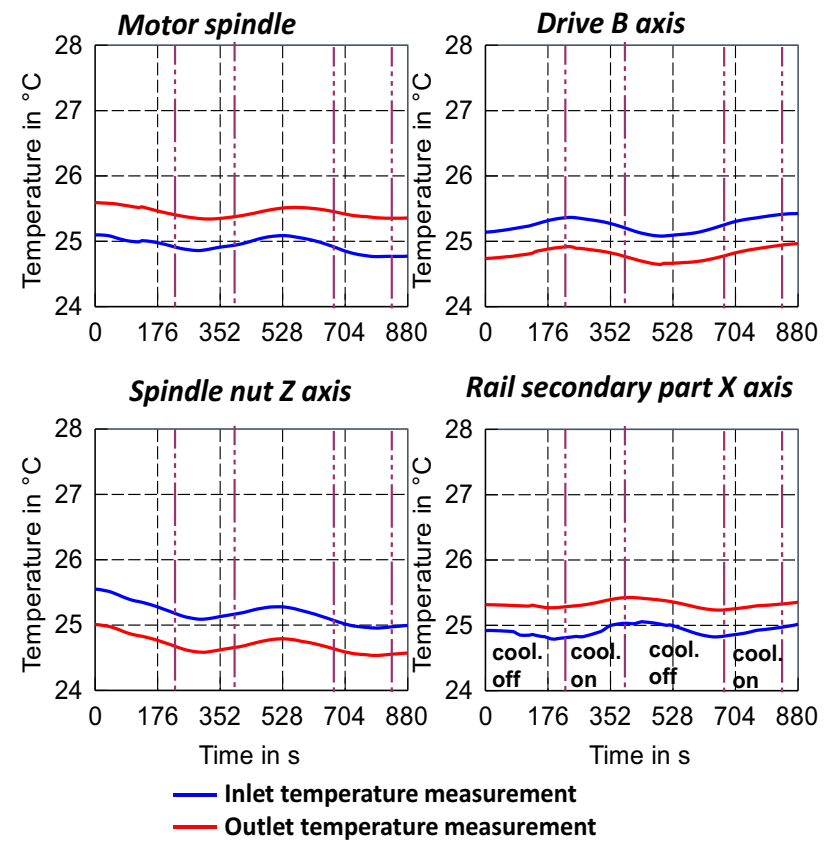

Figure 2. Temperature development in the manufactring process of DMU80. 


\section{System modelling and validation of current cooling design}

By using suitable simulation models, technical processes at the development stage can easily be visualized, examined, and optimized. The aim of modelling is to represent the technical process with sufficient precision and to generate well-founded results of the real process behaviour through subsequent calculations. By evaluating different process variants and parameters, it is possible to obtain extensive knowledge about the behaviour of a technical system without cost-intensive tests. The fundamental principle of the system modelling of cooling systems is based on thermo-hydraulic node-element modelling. With their help, different physical domains can be simulated in the simulation models. A brief overview of the physical domains and their relevant potential and flow quantities can be found in Table 1 as summarized [10].

Table 1. Physical domains and their respective flow and potential variables

\begin{tabular}{|c|c|c|}
\hline Physical domains & Potential variables & Flow variables \\
\hline Hydraulic & Pressure & Volume flow \\
\hline Pneumatic & Pressure & Mass flow \\
\hline $\begin{array}{c}\text { Mechanics } \\
\text { (translational) }\end{array}$ & Velocity & Force \\
\hline Mechanics (rotation) & Angular velocity & Torque \\
\hline Electric & Voltage & Current \\
\hline Thermal & Temperature & Heat flow \\
\hline
\end{tabular}

The nodes in the modelling represent the flow variable, which is also called capacitance. The elements are between the nodes as potential variables, which are considered as resistance. Fig. 4 illustrates the basic description of the thermo-hydraulic node-element model.

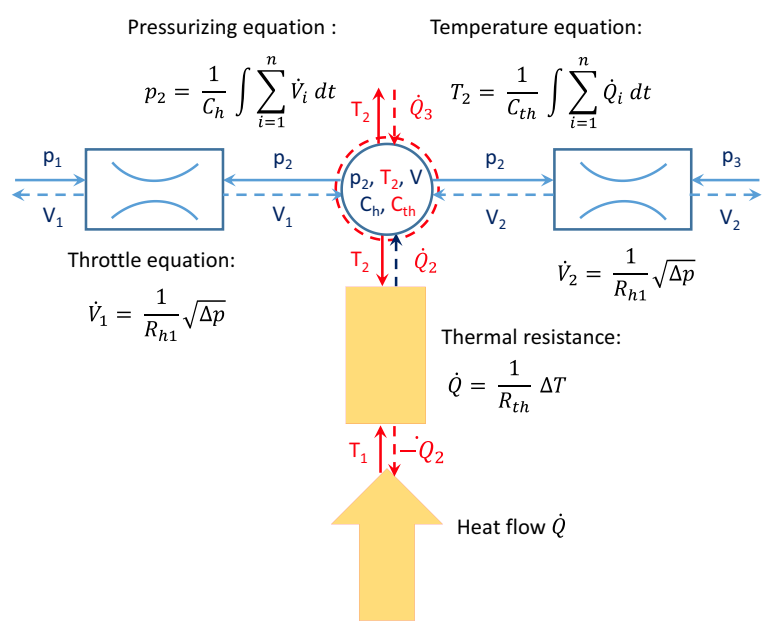

Figure 3. Basic description of the thermos-hydraulic nodeelement model.

The calculation of the thermal and the hydraulic resistances $R_{\text {th }}$ and $R_{\text {hy }}$ between two nodes is based on the following questions:

$$
\begin{aligned}
& R_{t h}=\frac{\Delta T}{\dot{Q}} \\
& R_{h y}=\frac{\Delta p}{\dot{V}}
\end{aligned}
$$

For the hydraulic and the thermodynamic domain, the laws of electrical engineering, such as Kirchhoff's circuit laws of series and the parallel connection of resistances can be used. In respect to Kirchhoff's node role

$$
\begin{gathered}
T=\frac{1}{C_{t h}} \int \sum_{i=1}^{n} \dot{Q}_{l} \cdot d t \\
p=\frac{1}{C_{h y}} \int \sum_{i=1}^{n} \dot{V}_{l} \cdot d t
\end{gathered}
$$

Furthermore, the heat transport through the cooling medium in the hydraulic pipe (forced convection), the heat transfer through the heat conduction in the pipe, and the heat transfer at the outer surface (free convection) of the pipe are taken into account, as exemplified in Fig. 5. Detailed information about the equations used for these three heat transfer mechanisms in the simulation model can be found in [11].

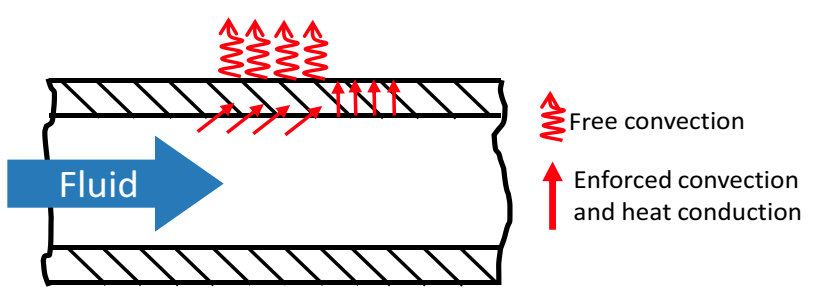

Figure 4. Heat transfer mechanisms at hydraulic pipe.

For the current cooling system of the DMU80, a simulation model is developed based on the modelling method and the machine documents. The simulation model of the cooling system mainly consists of a pump, flow valves, hydraulic pipes, a cooling unit, and the components as heat source. Each hydraulic connection is modelled by a hydraulic volume and a hydraulic resistance. As mentioned, the heat transfer between the pipes and the environment is taken in consideration. Fig. 6 shows the model structure of the cooling system implemented in the simulation. Table 2 shows the most important model parameters for the simulation model under consideration.

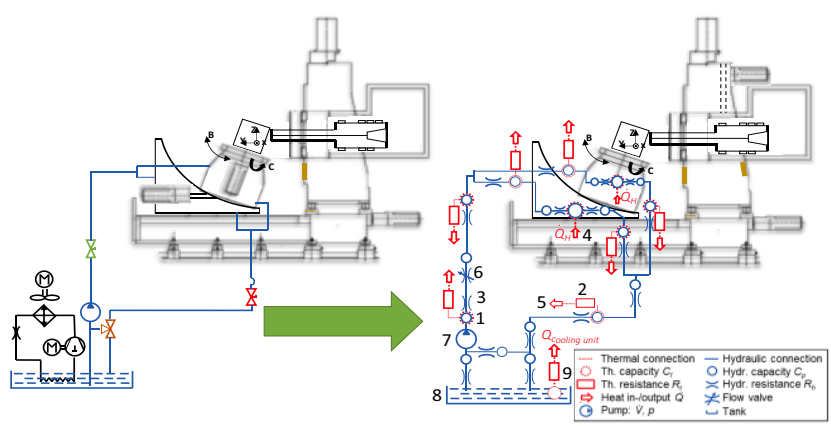

Figure 5. Model structure of cooling system in the simulation software. 
With the aid of the measured data, temperature, pressure drops and volume flow of each component, the heat flow of the respective components is calculated as in (5).

$$
\dot{Q}_{H}=\dot{V} \cdot\left[c \cdot \rho \cdot\left(T_{\text {outlet }}-T_{\text {inlet }}\right)+\left(p_{\text {outlet }}-p_{\text {inlet }}\right)\right]
$$

Table 2. Model parameter for the simulation model based on the model description in figure 6

\begin{tabular}{|c|c|c|}
\hline No. & Element & Parameters \\
\hline 1 & $\begin{array}{l}\text { Thermal or } \\
\text { hydraulic capacity }\end{array}$ & Geometry of the connections \\
\hline 2 & $\begin{array}{l}\text { Thermal } \\
\text { resistance }\end{array}$ & $\begin{array}{l}\alpha_{\text {inside }} \alpha_{\text {outside }} \alpha_{\text {conduction }} \text { fluid properties, } \\
\text { pipes properties, ambient properties }\end{array}$ \\
\hline 3 & $\begin{array}{l}\text { hydraulic } \\
\text { resistance }\end{array}$ & $\begin{array}{l}\text { Description form in the simulation } \\
\text { software, } \alpha(R e), \zeta(R e), \text { laminar } \\
\text { resistance, }(\Delta p, Q) \text { characteristic curve, } \\
\text { reference measurement }\end{array}$ \\
\hline 4 & Heat input & $\begin{array}{l}\text { With aid of measurement is calculated by } \\
\text { the equation ( } 4 \text { ) }\end{array}$ \\
\hline 5 & Heat output & $\begin{array}{l}\text { Calculated by forced convection, free } \\
\text { convection and heat convection of the } \\
\text { hoses, }\end{array}$ \\
\hline 6 & Flow valve & characteristic curve of the valve \\
\hline 7 & Pump & Flow rate and system pressure \\
\hline 8 & Tank & Tank capacity e.g. 201 \\
\hline 9 & Cooling unit & $\begin{array}{l}\text { Data sheets of the cooling unit, e.g. } \\
\text { cooling capacity } 4.5 \mathrm{~kW}\end{array}$ \\
\hline
\end{tabular}

For the modelling of the cooling system of the demonstration machine DMU80, a domain-crossing system simulation with a simulation program (SimulationX) is implemented. Fig. 7, Fig. 8, and table 3 depict a direct comparison of the simulation and measurement of the idle and the manufacturing process, respectively. The simulation model developed shows a high accuracy of the thermal (Fig. 7, Fig. 8) and the hydraulic quantities (Table 3 ) of the components under consideration. In the evaluation of the hydraulic quantities it is easy to see that the cooling system is characterized as a stationary system. In the contrast, the temperature profile of the components shows fluctuations over the entire process time. The simulation model is validated and thus can be used for the improvement of the current structure of the cooling system as well as for the development of process- and demand-oriented control strategies.

Table 3. Comparison of hydraulic quantities

\begin{tabular}{|l|l|l|l|l|}
\hline $\begin{array}{l}\text { Sub- } \\
\text { systems }\end{array}$ & $\begin{array}{c}\text { Measured } \\
\text { pressure in } \\
\text { bar }\end{array}$ & $\begin{array}{c}\text { Simulated } \\
\text { pressure in } \\
\text { bar }\end{array}$ & $\begin{array}{c}\text { Measured } \\
\text { flow in } \\
\text { l/min }\end{array}$ & $\begin{array}{c}\text { Simulate } \\
\text { d flow in } \\
\text { l/min }\end{array}$ \\
\hline $\begin{array}{l}\text { Motor } \\
\text { spindle }\end{array}$ & 3.3 & 3.4 & 9.3 & 9.2 \\
\hline $\begin{array}{l}\text { Drives B \& } \\
\text { C axis }\end{array}$ & 3.3 & 3.3 & 7.3 & 7.4 \\
\hline $\begin{array}{l}\text { Housing B } \\
\text { \& C axis }\end{array}$ & 3.3 & 3.4 & 9 & 9.1 \\
\hline XZ axis & 3.4 & 3.4 & 7.3 & 7.4 \\
\hline XY axis & 3.3 & 3.1 & 10.9 & 10.9 \\
\hline Pump & 4.5 & 4.6 & 43.8 & 44 \\
\hline
\end{tabular}

With regard to the above shown deficits of the current cooling system structure in the idle and the manufacturing process, the goal is to develop new structures for the cooling system to optimize its thermal behaviour and its effectivity according to the defined goal of a uniform temperature distribution at minimal energy consumption. Fig. 9 shows three new structures of a cooling system that can be applied for a demand-oriented supply. Controlled cooling systems based on the temperature development of the components enable an appropriate flow rate supply with an individually targeted temperature control at low energy consumption. The effectivity of the new structures will be evaluated first in regard to: a constant temperature behaviour at the 
components, a minimal pressure loss, and a minimal hydraulic power of the pumps.

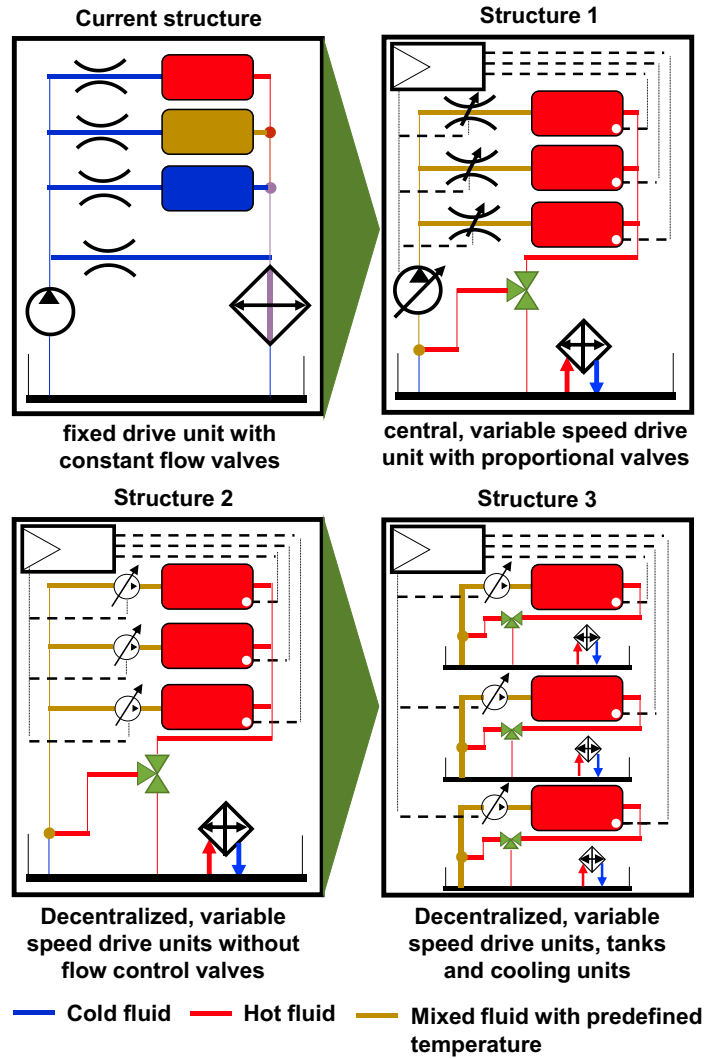

Figure 8. New structures for a cooling system.

\subsection{Structure 1}

The first examined cooling system structure, structure 1, is a central, variable speed drive unit with proportional valves. As shown in Fig. 10, this structure is presented by three components, the electrical cabinet, rotary table and motor spindle. Despite a central, variable drive unit, the components are cooled individually with the aid of a proportional valve. The system control of the cooling system compares the actual and the set temperature of the individual components, and, on this basis, adjusts the proportional valves as well as the central variable speed drive unit. The temperature detection is carried out via sensors in the components. If the temperature development of a component does not exceed a predefined threshold, the associated proportional valve will stay closed. Based on the measurement, an average equivalent heat flow in the idle process for each component is calculated. It is $150 \mathrm{~W}$ for the electrical cabinet and rotary table and $1500 \mathrm{~W}$ for the motor spindle. Only static operating points of the cooling system are considered in the simulation so that the thermal capacity of the components is not required. For the potential analysis of new cooling structures as well as the current cooling structure, three set temperatures are defined for the components: $26^{\circ} \mathrm{C}$ for the electrical cabinet, $27^{\circ} \mathrm{C}$ for the rotary table, and $28^{\circ} \mathrm{C}$ for the motor spindle. Furthermore, the system inlet temperature on the suction side of the pump is considered in the simulation at $25^{\circ} \mathrm{C}$.
The cooling unit stays in the two-point temperature control as bypass cooling and refers to the mixing temperature of the tank. The flow rate through a proportional valve can be calculated using the following equation:

$$
\dot{V}=B\left(y_{i}\right) \sqrt{p_{0}-p_{i}}
$$

The change of the supply pressure is described by the following equation:

$$
\dot{p}_{0}=\frac{K^{\prime}\left(p_{0}\right)}{V_{L, 0}}\left(\dot{V}_{\text {pump }}-\sum_{i=1}^{n} \dot{V}_{i}\right)
$$

In respect to the control strategy in Fig. 10, it can be seen, that the cooling structure under consideration has three control variables (component's temperatures) and four control elements (three proportional valves and a variable pump). This makes the system with an actual design concept over-determined. To solve this problem, three approaches can be taken into account [7]:

- Definition of a constraint

- Removal of a control element from the active control loop and

- Definition of an additional control variable.

Equations6 and 7 show the dependence of the individual flow rates on the components. Due to these correlations of the individual cooling circuits, the system considered is a so-called Multiple-Input-Multiple-Output System (MIMO system) and has cross-couplings. This means that one control element simultaneously influences several control variables.

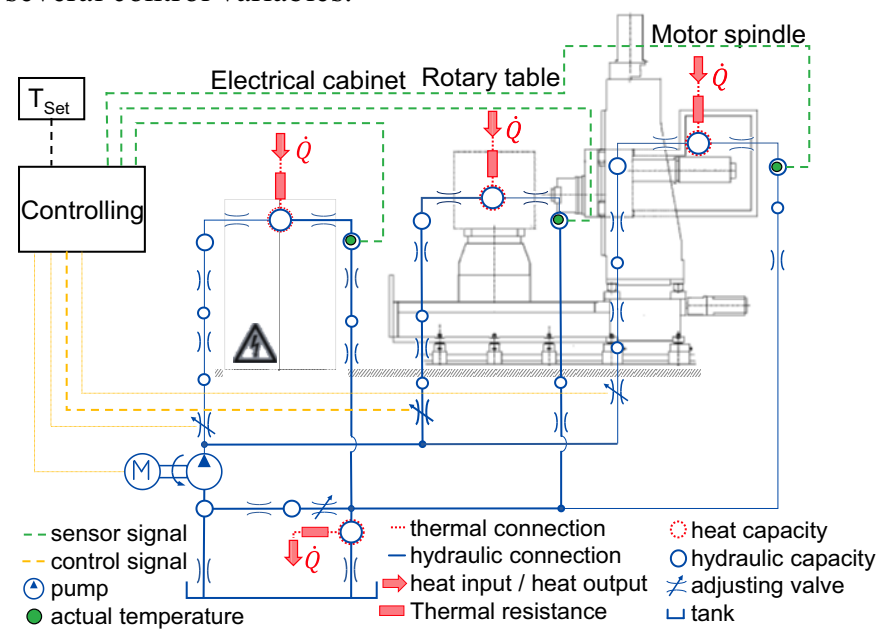

Figure 9. Control strategy of structure 1.

\subsection{Structure 2}

The second optimization structure of the cooling system structures under consideration shown in Fig. 11 is a decentralized, variable speed drive unit without flow control valves. In this structure, the components are also supplied individually but with the help of the variable speed pumps which are connected to a common tank and a cooling unit. This design does not require a proportional valve to distribute the cooling medium to the components. 
Exactly as in structure 1, the system control of the cooling system compares the actual and the set temperature of the individual components and on this basis adjusts the variable speed drive units. So, each pump supplies a different demand-oriented cooling volume flow. If the temperature development of a component does not exceed a predefined threshold, the pump remains inactive. Due to the individual supplies of each component with the decentralized pumps, the circuits operate independently. Therefore, this system consists of several in-dependent Single-Input-SingleOutput control systems (SISO system). Consequently, each temperature can be regulated by a PI-controller. The I-term of the controller eliminates any remaining control deviation of the P-term. The system boundary condition of structure 1 applies also to structure 2 in regard to the components' set temperature, heat input, system inlet temperature and static operating points of the system, and the cooling unit.

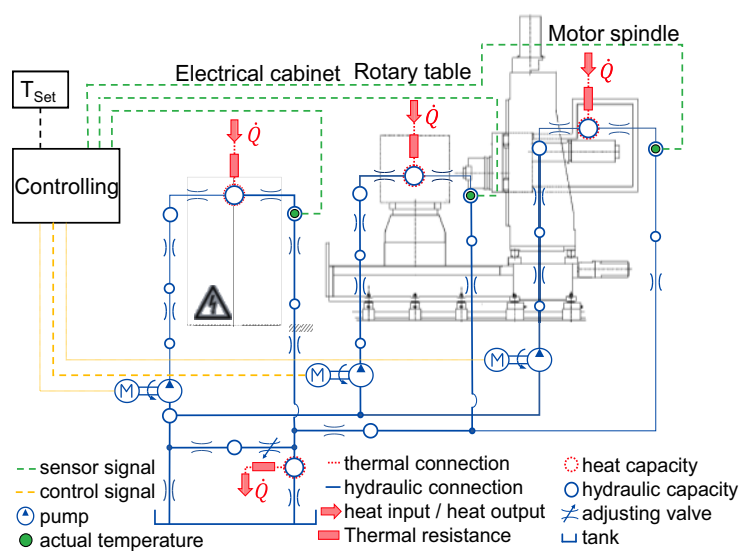

Figure 10. Control strategy of structure 2.

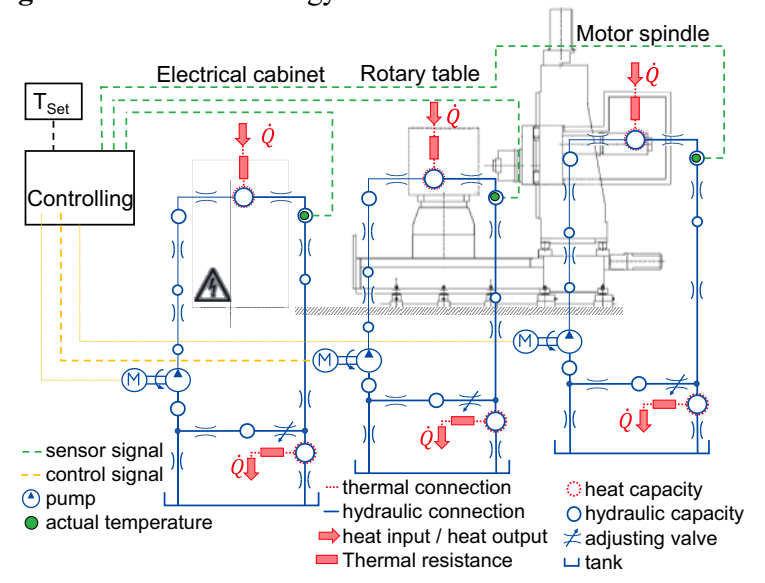

Figure 11. Control strategy of structure 3 .

\subsection{Structure 3}

The system design of structure 3 is a decentralized system. Each circuit in the system has a variable speed drive unit, a tank and a cooling unit. Exactly as in structure 2, this structure does not need any flow control valves. The system control of the cooling system compares the actual and the set temperature of the component and on this basis adjusts the variable speed drive units. The main idea for investigating this structure in Fig. 12 consists of completely separate cycles. This makes it possible to reduce the line lengths, especially for large processing machines, by placing the respective cooling system as close as possible to the component to be cooled. Another advantage of cooling system structure 3 is the possibility foe cooling the different components with different cooling media. This is not possible with cooling system structures 1 and 2 through the common tank.

\subsection{Results and evaluation}

The evaluation of the results should begin with the main task, the control of temperatures of the cooling system. As a result of the simulation models, for all three investigated system structures, the set temperatures are kept within the actual temperature limits. The calculated temperature development of the electrical cabinet, rotary table, and motor spindle are shown in Fig. 13 as a function of the heat input. The set temperatures are indicated by a dashed characteristic curve coloured light blue, the calculated actual temperatures by a continuous characteristic curve coloured orange and the temperatures of the current system structure by using a characteristic curve coloured purple. A comparison of the temperature curves of the three controlled system structures and the current system highlights two differences. Firstly, by using a feedback control system, the temperatures achieved are constant in all cooling circuits, in contrast to the current cooling system. This can be traced back to the adaptation of the cooling volume flows to the respective cooling requirement by the control unit. The second difference is the ability to adjust the temperature level of the respective component by setting the set temperatures on the control circuit. This is not possible with the current cooling structure: as a result of this system behaviour, the actual temperatures increase with the increasing heat input in the current cooling system. In addition to the circuits of the electrical cabinet and the rotary table, the dependence of the actual temperature on the thermal load (heat input) has a particularly significant effect on the circuit of the motor spindle. Here, the component temperature fluctuates between 27 and $29{ }^{\circ} \mathrm{C}$. In contrast, constant component temperatures can be achieved in all circuits with the three controlled cooling system structures. For all controlled cooling system structures, there is a slight deviation of $0.2{ }^{\circ} \mathrm{C}$ between the actual and the set temperatures. This control deviation is due to the required control difference in the control loop. In the diagram of the volume flows, bottom right in Fig. 13, the reduced cooling volume flows of the controlled cooling system structures are to be noticed in comparison to the current system structure. In the cooling circuits of the rotary table and the electrical cabinet, the volume flows are reduced from $12 \mathrm{l} / \mathrm{min}$ in the current system to 2 to $2.5 \mathrm{l} / \mathrm{min}$ or 2.5 to $5 \mathrm{l} / \mathrm{min}$. This corresponds to an averaged reduction of about $80 \%$ in the cycle from the rotary table and about $70 \%$ in the circuit of the electrical cabinet. The cooling volume flow rate of the current 
system is $12.5 \mathrm{l} / \mathrm{min}$ in the main spindle circuit and between 8 and $161 / \mathrm{min}$ in the new cooling system structures. It can be ascertained that the volume flow control based on the temperature development is a means for designing the system in a more energy-efficient way based on each individual component's demand. In principle, it is not essential for the temperature behaviour whether the flow rate is set by a controlled pump in each circuit, as in structure 2 and 3 or by a controlled pump and proportional valves, as in structure 1. For simplification, the diagrams of the three system structures have therefore been summarized in Fig 13.
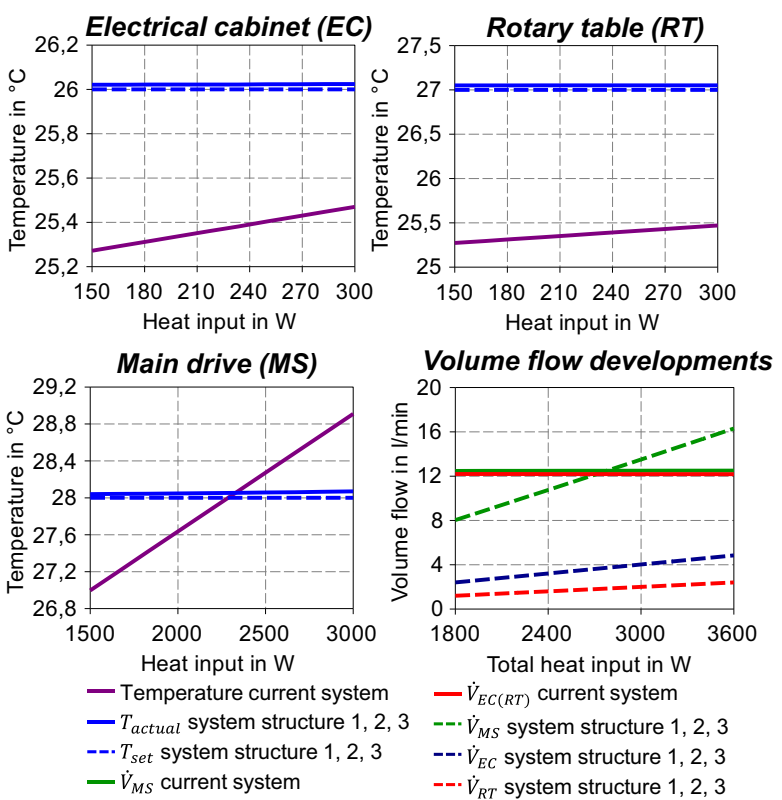

Figure 12. Simulation results of new cooling structure under consideration in comparison to current cooling structure.

The second objective of the study of different cooling system structures is to show a comparison of the required hydraulic power and the respective energy requirements of these systems with the current cooling system structure. The total hydraulic power of the pump in the current cooling system structure of two demonstration machines (DBF630 \& DMU80) and structure 1, 2, and 3 for different heat inputs is pointed out in Fig. 14. With the variable central displacement pump (structure 1), the total hydraulic power is approximately $160 \mathrm{~W}$ at maximal heat input. Similar to structure 1, the total hydraulic power of the variable speed displacement pumps in structure 2 and structure 3 is about $120 \mathrm{~W}$ and $110 \mathrm{~W}$ at maximal heat input, respectively. Compared to this, the hydraulic power of the fixed displacement pump (current structure) of DBF630 amounts to $370 \mathrm{~W}$ (40 1/min at $5.5 \mathrm{bar}$ ) and of DMU80 to $340 \mathrm{~W}(45 \mathrm{l} / \mathrm{min}$ at $4.5 \mathrm{bar})$. A significant energy savings of $56.7 \%$ to $53 \%$ in structure 1 in contrast to the current structures of DBF630 and DMU80 are possible. Similar to structure 1, the energy savings of $67 \%$ to $64.7 \%$ for structure 2 and $70.5 \%$ to $67.6 \%$ for structure 3 in contrast to the current structures of DBF630 $(370 \mathrm{~W})$ and DMU80 $(340 \mathrm{~W})$ can be shown. The new cooling system structures under consideration are significantly more energy-efficient compared to the current cooling system structures of DBF630 or DMU80 with a continuous cooling volume flow.

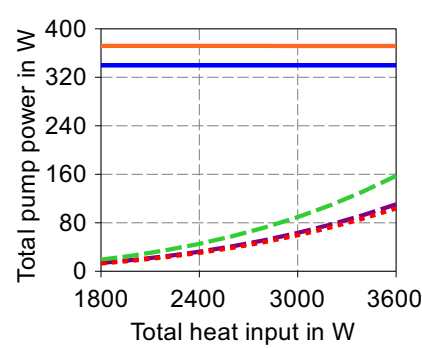

- Phydr. DBF $630--P_{\text {hydr. Structure } 2}$
- Phydr. DMU80 - - Phyr. Structure 3
- - Phyr. Structure 1
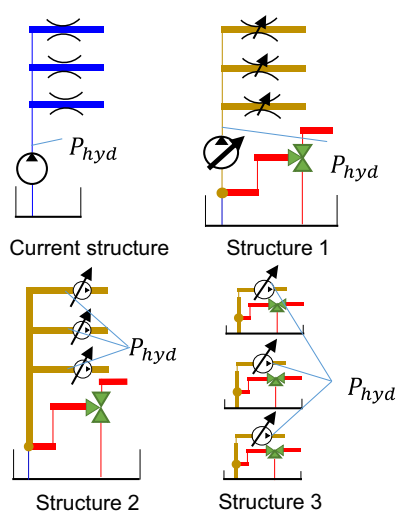

Figure 13. Comparison of the pump performance in the current and in the new cooling system structure.

\section{Summary and outlook}

The experimental investigation of the cooling system of a demonstration machine has been instrumental in determining the effectiveness as well as the energy consumption of the cooling system [9, 12]. It could be shown that the machine components are not cooled specifically with the current cooling structures, and that cooling is adjusted insufficiently in reference to the component's demand and process requirement. Therefore, the investigation and evaluation of new cooling concepts, both simulative (network models) and experimentally (test rig), is of great importance.

The new cooling structures examined in this paper, central, variable speed drive unit with proportional valves (structure 1), decentralized, variable speed drive units without flow control valves (structure 2) and decentralized, variable speed drive units, tanks and cooling units (structure 3), show high accuracy with respect to the temperature control of the components compared to the current cooling structure. Apart from this, the hydraulic pump performance of the new structures is about $53 \%$ to $70.5 \%$ lower than the hydraulic pump of the current cooling structures.

The focus of further research of the projects will firstly address an energetic analysis of the overall system for each structure considered, the energy consumption of the electrical motor, frequency converter etc. Secondly, the new structures under consideration shall demonstrate their benefits in practice and not only in simulation. To this end, a test rig is being developed which will allow an experimentally sound statement about the structures regarding their effectiveness and efficiency.

\section{Acknowledgment}

The research activities presented are part of the project "Thermo-energetic description of fluid systems" (Ref. No. CRC/TR 96, A04). The authors would like to thank the 
German Research Foundation (DFG) for financial support. Funded by

\section{D $=\begin{aligned} & \text { Deutsche } \\ & \text { Forschungsgemeinschaft }\end{aligned}$}

\section{References}

1. K. Großmann, O. Gritt, Introduction. In: K. Großmann editor. "Thermo-energetic Design of Machine Tools", Heidelberg: Springer-Verlag; p.112, (2015).

2. C. Brecher. "Effizienzsteigerung von Werkzeugmaschine durch Optimierung der Technologien zum Komponentenbetrieb“-EWOTek, Apprimus Verlag, Aachen, (2012).

3. B. Denkena, T. Garber, "NCplus Prozess und wertschöpfungsorientiert gesteuerte Werkzeugmaschinen“, PZH Verlag, (2013).

4. U. Götze, H.J. Koriath, A. Kolensikov, R. Linder, J. Paetzold, C. Scheffler. "Energetische Bilanzierung und Bewertung von Werkzeugmaschinen“, Tagungsband Energieeffiziente Produkt- und Prozessinnovationen in der Produktionstechnik eniPROD, Chemnitz, (2010).

5. L. Shabi, J. Weber, J. Weber. "Modellgestütze Potentailanalyse eines dezentralen Fluidsystem" in 5. Kolloquium zum SFB/TR96, Thermo-energetische
Gestaltung von Werkzeugmaschinen- Modellierung und Simulation, Chmentz, March 22-23, (2017).

6. Sauter Group Product brochures Ventile und Antriebe, P100014406 - V2, www.sautercontrols.com; 07.03.2018; 09:13.minutes.

7. J. Popken, L. Shabi, J. Weber, J. Weber, "System Modelling and Control Concepts of Different Cooling System Structures of Machine Tools", In: first CIRP Conference on Thermal Issues in Machine Tools, Dresden, Germany, March 21-23, (2018).

8. International standard ISO 230-3. Test code for machine tools, part 3: Determination of thermal effects. Second edition (2007-08-15).

9. L. Shabi, J. Weber, J. Weber, "Analysis of the Energy Consumption of Fluidic System in Machine Tools", In: 50th CIRP Conference on Manufacturing Systems, Taichung, Taiwan, May 3-5, (2017).

10. Institute of Fluid Power, lecture script of Modelling and Simulation of Fluidic Systems. Prof. Dr.-Ing. J. Weber, (2017).

11. L. Shabi, J. Weber, J. Weber, "Investigation of the Potentiale of Different Cooling System for Maschine Tools“ In: 11th Internation Fluid Power Conference, Aachen, March 19-21,(2018).

12. L. Shabi, J. Weber, J. Weber, "Model-based Analysis of Decentralized Fluidic Systems in Machine Tools" In: 15th Scandinavian International Conference on Fluid Power, SICFP'17, Linköping, Sweden, June 79, (2017). 